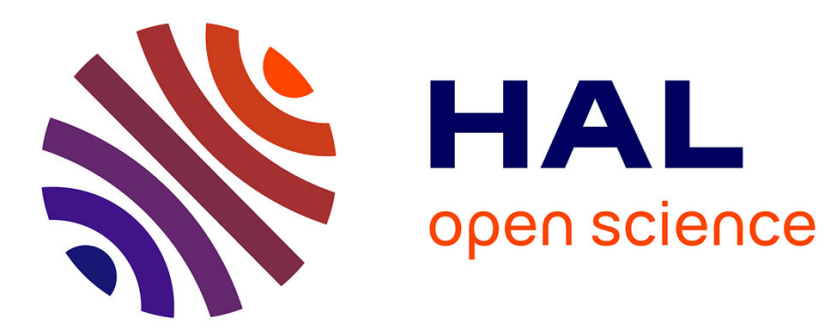

\title{
L'image au défi de sa propagation virale
}

Pierre Morelli

\section{To cite this version:}

Pierre Morelli. L'image au défi de sa propagation virale. Colloque international L'image à l'épreuve de la viralité, Institut Supérieur des Arts et Métiers de Mahdia (Tunisie), Nov 2016, Mahdia, Tunisie. pp.7-32. hal-01721612

\section{HAL Id: hal-01721612 \\ https://hal.univ-lorraine.fr/hal-01721612}

Submitted on 24 Jun 2018

HAL is a multi-disciplinary open access archive for the deposit and dissemination of scientific research documents, whether they are published or not. The documents may come from teaching and research institutions in France or abroad, or from public or private research centers.
L'archive ouverte pluridisciplinaire HAL, est destinée au dépôt et à la diffusion de documents scientifiques de niveau recherche, publiés ou non, émanant des établissements d'enseignement et de recherche français ou étrangers, des laboratoires publics ou privés.

\section{(이) $\$$}

Distributed under a Creative Commons Attribution - NonCommercial - NoDerivatives $\mid 4.0$ 


\section{Pierre MORELLI}

«L'image au défi de sa propagation virale»

Éditorial de Dialogue des révolutions : LA VIRALITÉ

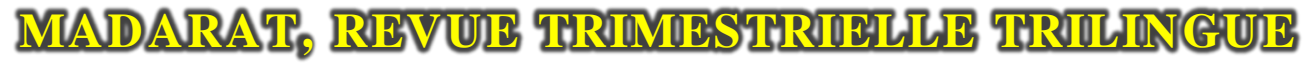 MOULTIDISCIPLINANORE}

N²9-30 : été - printemps 2017

Association Madarat Maarifia

BP. 243 La manouba 2010 Tunisie

associamadarat@gmail.com

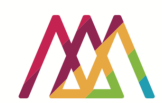

ISAMM Actes du colloque international : L'image à l'épreuve de la viralité. Organisé par l'Institut Supérieur des Arts et Métiers de Mahdia (Tunisie) les 18-19-20 novembre 2016.

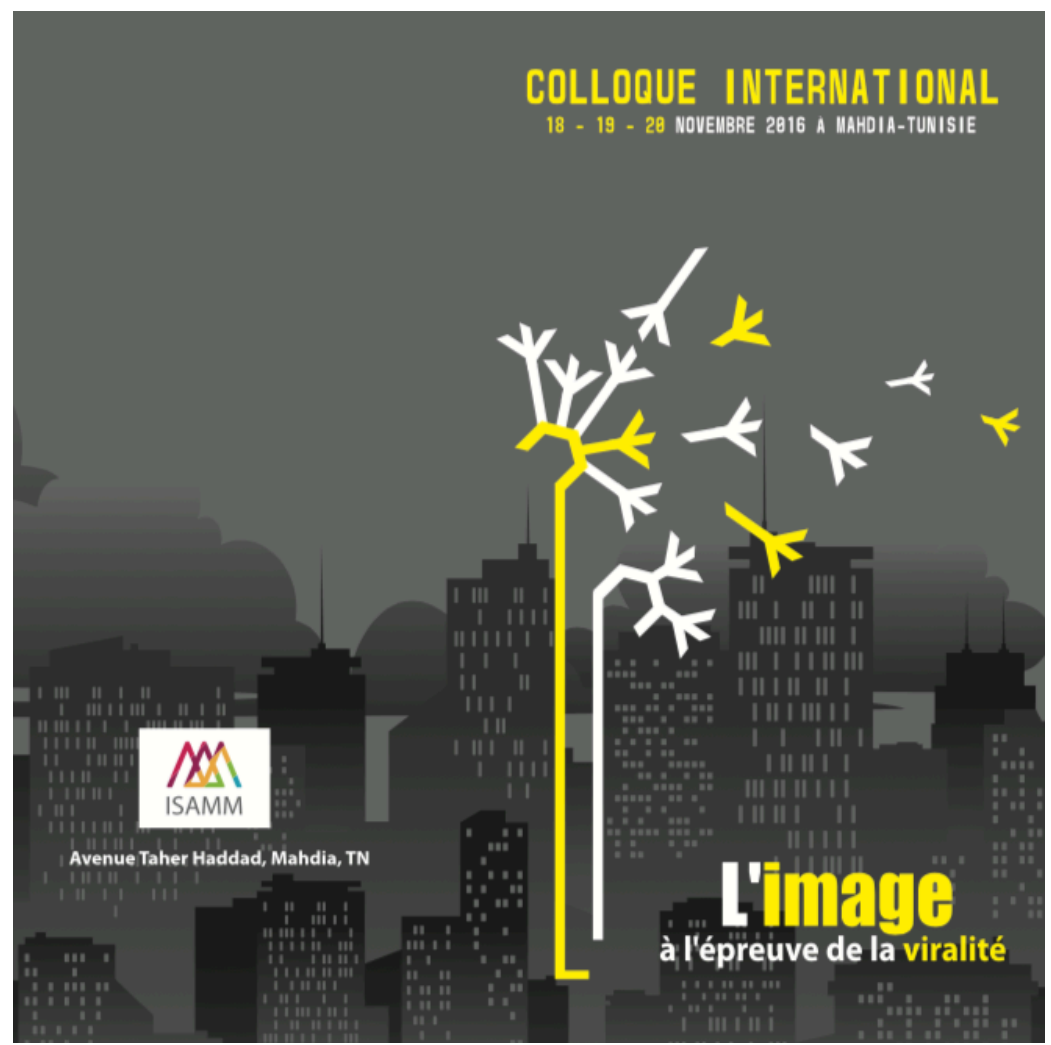

Pour citer ce chapitre :

Morelli P. (2017). L'image au défi de sa propagation virale, Madarat, n²9-30. Dialogue des révolutions : la viralité, 7-32.

Ce document est mis à disposition selon les termes de la licence Creative Communs : attribution, pas d'utilisation commerciale, pas de modifications 3.0.

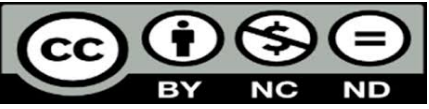




\title{
L’IMAGE AU DÉFI DE SA PROPAGATION VIRALE
}

\author{
Pierre MORELLI \\ Maître de conférences \\ Centre de recherche sur les médiations \\ Université de Lorraine \\ pierre.morelli@univ-lorraine.fr \\ http://crem.univ-lorraine.fr/morelli-pierre
}

Avec l'intensification des modalités d'interconnexion qu'offrent les réseaux numériques, l'image est de nos jours confrontée à de nouveaux défis qui l'amènent à redéfinir, à l'aune des contextes contemporains, la fonction de représentation qu'elle assume depuis toujours à des fins de monstration (présenter, montrer) et de figuration (symboliser, signifier) au sein de conditions de production et de diffusion nouvelles.

Si le concept de représentation se construit, rappelons-le, dans trois sphères de sens, la « figuration », la "substitution » et la « répétition » (Morelli, 2006), l'image incarne, le plus souvent, un sujet qu'elle représente et dont elle pallie l'absence. Cette incarnation opère parfois selon un principe de réitération offerte au destinataire ou laissée à son initiative sur la base d'une volonté d'action, ou de choix navigationnels, lesquels contribuent à différencier chaque « répétition » donc chaque re-présentation. La « figuration » concerne alors la mise en œuvre de mondes qui constituent autant de schémas perceptifs imitant la réalité. Devenue par nature numérique, lieu et objet de l'action, l'image s'articule avec divers types de véhicules sémiotiques (textes, images et sons) au sein des réseaux et se place plus que jamais «sous le signe du circuit court ${ }^{1}$, renforçant, à force d'actualisations résultant des actions des internautes, l'idée de substitution d'une réalité absente.

Opérant, de manière intriquée à la fois sur le registre de la médiatisation et sur celui de la médiation, tant dans sa fabrication que dans sa diffusion donc dans sa réception, l'image est de nos jours soumise à plusieurs types de temporalités qui coexistent et s'hybrident. Elle nous amène tout d'abord à vivre l'expérience du temps continu de la programmation médiatique qui, avec la radio puis avec la télévision, délocalise les conditions de réception et nourrit une présence "ubiquitaire" et personnelle. Distribuée à tous les téléspectateurs en même temps, l'image télévisuelle parvient en effet directement au domicile du téléspectateur via une transmission hertzienne en direct, ce qui amène le public à renouer avec la relation de continuité propre à l'entropie de la vie humaine, perdue dans le cinéma ${ }^{2}$ et intimement liée à la notion de présentation telle que la définit de Paul Virilio (Forest, Virilio, $1988: 15$ ) à savoir une « révélation ».

Cette expérience s'articule de plus en plus avec une pratique a priori antagoniste, celle du temps discontinu offerte par la rediffusion de programmes. Introduite par le câble, cette pratique a vu son succès s'amplifier à travers deux modèles de diffusions complémentaires

\footnotetext{
${ }^{1} \mathrm{Et}$, prolongeant le mouvement apparu avec le développement de la vidéosphère, donc du direct médiatique, l'image : « promeut l'abrégé, (... et) la transitivité maximale. À quoi bon des procès en bonne et due forme, en des lieux solennels, selon des procédures compliquées ? Oubliez ces rituels surannés, faites rentrer les cameras, et chacun tranchera selon son cœur, la couleur de peau ou le compte en banque de l'inculpé. À quoi bon des professeurs et des disciplines ? Ouvrez les écoles, mettez-y des animateurs et de bonnes émissions, et les enfants trouveront d'eux-mêmes leur équilibre personnel. Justice, éducation, politique, beaux-arts - partout résonne l'injonction : "collez donc à la vie". Nous voilà sommés de préférer le flux à la forme, le jaillissement à l'élaboration, le primaire au secondaire, le regard de plongée (participatif, fusionnel) au regard de surplomb (critique, objectivant). » (Debray, 1996).

2 À travers le montage, le cinéma prend bien des libertés avec le temps entropique (ellipses, flashback...).
} 
dans les médias numériques contemporains : le streaming et le podcasting. Entre direct et rediffusion de programmes enregistrés s'intercale alors une nouvelle forme temporelle qui ouvre une troisième voie entre production et réception: le temps réel des technologies numériques. Avec l'avènement et la généralisation du temps réel du numérique, donc avec la mise en œuvre du temps de l'instantanéité introduit et produit par l'interactivité (Couchot, 1997) « l'imageur » et le « regardeur » sont alors placés dans un rapport de coïncidence entre le temps du «faire » et celui du « voir » puisqu'en effet « la création de l'image dépend de l'action directe du regardeur, donc de sa nécessaire et immédiate présence » (Couchot, 2007 : $50)$.

Toutes ces différentes temporalités sont de plus en plus appelées à s'hybrider, entrainant les pratiques en ligne dans une logique d'accélération du temps et des temporalités (Rosa, 2005), source de désynchronisation entre les acteurs sociaux et les institutions tenues d'accompagner le changement. L'image n'est pas pour autant uniquement entrainée dans une fuite en avant incontrôlable puisque cette accélération temporelle l'amène à s'inscrire dans un temps long et d'une certaine manière dans un temps cyclique (Gui Ekwa, 1995), offrant de surcroit à la représentation des potentialités de réitération décalées dans le temps.

$\mathrm{Au}$ sein des réseaux numériques, cadres contemporains privilégiés pour la diffusion et l'échange de l'image, la question du sens véhiculé revient en force avec le développement d'une intention narrative qui accompagne le cycle de vie de l'image, intention que Louis Marin et Jean Verrier (2017) prêtent aux $\operatorname{artistes}^{3}$ et qui s'affirme désormais à force d'échanges iconographiques qui innervent les relations interpersonnelles et les médias numériques (réseaux sociaux, réseaux $3 \mathrm{G}$ et $4 \mathrm{G}$, sites web...). Foisonnante, cette mise en circulation d'images concourt également à faire coexister, dans le quotidien des internautes, une volonté, voire un impérieux besoin de représenter l'absence à travers la présence sans cesse réaffirmée et envahissante de l'image, relayée, annotée, déclinée voire détournée. Ainsi, l'image constitue-t-elle aujourd'hui à la fois l'objet et le terrain d'exercice de la circulation d'informations à travers les réseaux d'information et de communication. Toutefois, mise au défi de sa propagation virale, l'image numérique est, allons nous le voir, bien plus qu'une image.

\section{L'image à l'ère des réseaux numériques est bien plus qu'une image}

À l'ère des réseaux numériques, l'image est bien plus qu'une image livrée au regard. Elle s'affirme comme objet et sujet de questionnements et préfigure l'action.

\section{L'image comme objet et sujet de questionnements}

À travers les aphorismes qu'il distille dans ses films ou qu'il aime à prononcer en interview le cinéaste Jean-Luc Godard construit une vision personnelle du cinéma en tout point opposée à la télévision qui a ses yeux n'aurait au mieux pour vertu que la faculté de transmettre : « au cinéma on peut ne rien faire tout en faisant quelque chose de ce rien... par contre la télévision ne fait rien, la télévision c'est avant tout transmettre, il faut répéter, travailler avant voire et après faire quelque chose $»$. De plus, entre grand écran et petite lucarne les enjeux sont pour lui de natures opposées puisque, si « La télévision fabrique de l'oubli. Le cinéma fabrique des souvenirs. », et que « Quand on va au cinéma, on lève la tête. Quand on regarde la télévision, on la baisse. ». Cependant, pour Jean-Luc Godard, la différence entre cinéma et télévision tient aussi et surtout au rapport que ces deux entités entretiennent au visible et donc à l'image.

\footnotetext{
${ }^{3}$ « Toute représentation narrative met donc en jeu trois éléments fondamentaux : d'une part, les actions des acteurs du récit; d'autre part, le temps et les moments de l'histoire racontée ; enfin, l'espace et les lieux où ces actions représentées prennent place. » (Marin, Verrier : 2017).
} 
Au cinéma, insiste le réalisateur, l'image a pour vertu de mettre en scène l'invisible, ce qu'il énonce en ces termes : «Il y a le visible et l'invisible. Si vous ne filmez que le visible, c'est un téléfilm que vous faites. ». Cet invisible c'est aussi et surtout le sens que le spectateur infère et construit du film qu'on lui propose. C'est, d'une certaine façon, ce qui sépare " présentation » (régime sur lequel opère la télévision) et " représentation » avec toutes sortes de données contextuelles à destination des publics (didascalies...), la représentation ayant notamment pour intérêt la restitution, tout au moins partielle de cet « invisible ».

Qu'elle engage une position réflexive ou émotionnelle, l'image ainsi conçue de manière cinématographique amène à prendre du recul par rapport à l'immédiateté de la réalité que toute image affiche ou représente. L'image de télévision et celle de cinéma se distingueraient donc par l'intention et la finalité qui président à leur création. De plus, selon Jean-Luc Godard l'image au cinéma ne serait ni figée ni définitive. Figure emblématique de "la nouvelle vague » ce réalisateur renvoie l'image au statut d'élément simple de sa grammaire cinématographique, affichant dans le film Vent D'est (1970) un carton qui annonce : «Ce n'est pas une image juste, c'est juste une image ». Pareille assertion rompt toutefois avec l'idée de puissance de l'image au cinéma que dix ans plus tôt, Godard affirmait, dans le film Le Petit soldat (1960) que "La vérité au cinéma, c'est 24 images par seconde ». Ces deux prises de position à dix ans de distance montrent combien la question du statut et de l'essence de l'image le hante.

À l'ère de l'information numérique en ligne, alors que les images inondent les canaux de communication et qu'elles accompagnent le quotidien de chacun, au même titre que le texte et le son, l'image doit être pensée comme composant discursif, donc comme objet sémiotique choisi et/ou construit au service de la production de messages, voire comme le corps du message lui-même, sa forme et sa portée perlocutoire variant en fonction du contexte.

Interrogeons l'image numérique, non pas comme dispositif (au sens foucaldien), à savoir l'art de tisser un réseau entre des éléments hétérogènes (dits \& non-dits) afin de structurer les rapports de force et les relations de pouvoir, mais comme moyen permettant la représentation de la réalité contemporaine, donc comme appareil aidant à configurer la sensibilité commune (Déotte, 2004) et qui d'une certaine manière hybride aujourd'hui "présentation » et « représentation ».

\section{L'image du regard à l'action}

Les appareils dont il est question ici affectent grandement la perception contemporaine. Les images produites par ordinateur ne sont pas faites pour être vues précisait Jean-Louis Weissberg à l'occasion d'une communication prononcée lors du colloque Vers une culture de l'interactivité, tenu à Paris en 1988 à la Cité des sciences et de l'industrie de La Villette. Cette affirmation visait non seulement à produire un effet sur l'auditoire et plus tard sur le lecteur car, sauf à parler d'image mentale, toute image est bien évidemment, à un titre ou à un autre, amenée à être vue ${ }^{4}$. Cet aphorisme insiste sur le fait que le traitement numérique de l'image participe d'un glissement de la question du visible vers une problématique du lisible. L'ingénieur qui conçoit et dessine une aile d'avion par ordinateur cherche en effet moins à soigner l'apparence de la représentation visuelle, qu'à montrer sous tous ses angles l'objet représenté et dans la pluralité de formes qu'il peut prendre à des fins compréhensives, donc à créer un modèle fonctionnel. Le cadre de fonctionnement (Flichy, 1995) de l'avion consiste en effet à créer une dépression sur le bord de l'aile, afin de produire une poussée verticale opposée à la gravitation et qui augmente avec la vitesse linéaire de l'appareil. Étant plus lourd

\footnotetext{
${ }^{4}$ Sauf dans le cas des réalisateurs des premiers projets de film en images de synthèse qui n'ont pu voir le résultat, faute de disposer d'outils suffisamment puissants.
} 
que l'air, l'avion ne peut en effet pas utiliser la poussée d'Archimède pour s'élever. Désormais, derrière la conception de tous types d'avions se nichent des modèles mathématiques reproduisant les tests en soufflerie afin d'optimiser l'écoulement de l'air sur le fuselage et de visualiser les parties sur lesquelles opère la sustentation. Opérant sur le registre scientifique que l'imagerie médicale dont la finalité est moins esthétique qu'informationnelle, les images produites par ordinateur sont avant tout intelligibles. L'image devient alors un intermédiaire donnant accès à l'information et en cela constitue un matériau livré à la sagacité du spécialiste qui y prélève du sens.

La technologisation qui saisit l'image produit en outre des effets sur la perception en général et sur la dimension visuelle qu'elle représente en particulier. Dans le même article Jean-Louis Weissberg (1988: 53) proclame que «Les technologies qui prothèsent le regard en reformulent les conditions d'exercice ». La vision du monde serait donc appareillée par les technologies contemporaines et la culture qui prend forme suite au développement de leur utilisation. Pareil appareillage ne peut toutefois véritablement opérer qu'à condition de mobiliser les moyens techniques contemporains. Constatant que la réalité change, notamment sous l'impulsion des technologies contemporaines, Paul Virilio (1988: 14) précise en effet que cette "réalité en mutation» ne peut plus être toutefois représentée avec les outils traditionnels :

\footnotetext{
«...le monde ne pourra plus se représenter désormais à travers une sculpture, ou l'image fixe du tableau, il ne pourra trouver sa juste "représentation" que dans la vitesse du mouvement, la juxtaposition des sources d'informations, l'hétérogénéité simultanée de ses supports physiques et électroniques ».
}

Ainsi, en réponse aux besoins générés par les contextes émergents, la technologie construitelle des appareils au service d'une vision renouvelée des rapports sociaux contemporains. Les technologies, précise Jean-Louis Déotte (2004 : 106 et 109), déterminent en effet le mode même d'apparition des événements et inventent des temporalités et des spatialités propres à chaque époque. À travers les technologies s'élaborent donc des représentations qui déconstruisent les relations spatio-temporelles tant sur le plan formel qu'en profondeur.

L'histoire de l'art nous rappelle par ailleurs qu'à chaque période correspond une modélisation de la perception. Ainsi, lorsqu'il, met en œuvre les principes généraux de la perspective dont la paternité est attribuée au mathématicien Ibn al-Haytham, l'architecte florentin Filippo Brunelleschi formalise un nouveau système de représentation du monde qui appareillera par la suite le regard et qui va très fortement inspirer et influencer les peintres de la renaissance. Toute une série d'appareils projectifs vont à la suite de la perspective être progressivement être mis au point. Citons tout d'abord la camera obscura, dont le principe décrit par Aristote et qui sera mise en application dès le $16^{\circ}$ siècle, puis la photographie au $19^{\circ}$ puis le cinéma.

Aujourd'hui les appareils auxquels nous avons affaire pour modéliser et intelliger la complexité contemporaine sont les réseaux numériques et l'interactivité qui les traverse. Une modélisation artistique des activités menées sur les réseaux et proposée par Jean-Louis Boissier (2004) retiendra, à ce propos, toute notre attention. Partant de l'idée de perspective cette proposition théorique interroge la dimension interactive qui se niche dans les réseaux, amenant d'une part l'usager à en faire l'expérience et constituant d'autre part un marqueur propre à l'époque contemporaine. La dimension visuelle que contribue à construire la perspective optique est en effet un préalable nécessaire mais non suffisant pour saisir et modéliser les relations à l'œuvre dans l'interactivité. L'artiste/chercheur (ibid.) invite alors à penser l'interactivité selon trois composantes indépendantes qui, réunies, composent ce qu'il choisit d'appeler la «perspective interactive ». De la même manière que trois dimensions spatiales (l'abscisse, l'ordonnée et la côte) fondent la perspective optique pour Jean-Louis Boissier il est possible d'identifier trois composantes propres cette fois ci, non à l'espace tridimensionnel mais à l'interactivité : la visibilité, la lisibilité, et la jouabilité. Relevant 
également de questions propres à l'optique optique, la perspective de la visibilité participe de l'affichage de l'espace virtuel tridimensionnel ${ }^{5}$ et anticipe l'action qui sera menée dans cet espace étant à même "de saisir, de déduire des interactions, de décrire les interactions " (ibid. : 266). L'apparition de l'espace calculé par ordinateur amène dans une autre dimension à interroger la « lisibilité de l'espace symboliquement reconstruit, mais aussi (la) lisibilité du texte, (et) du paysage textuel en réseau » (ibid. : 263) et, via « le jeu des désignations, qui fonde l'essentiel des programmes interactifs sur écran » (ibid. : 268), construisant alors une profondeur sémantique. Le troisième volet de la modélisation proposée par Jean-Louis Boissier concerne l'interactivité en soi et plus précisément les actions menées dans les espaces relationnels afin d'en assurer la jouabilité

Le principal avantage consiste à proposer ici une modélisation dynamique des interactions produites par et au sein des réseaux et de la pulsion info-communicationnelle qui saisit le monde contemporain. Remarquons qu'en affirmant la place centrale de l'interactivité et du numérique dans la complexité contemporaine, ces propositions revisitent et actualisent le débat qu'ont entretenu par le passé l'art et la science l'impact de ces différents appareils concernera et que mêlant l'interactivité et la puissance du numérique les réseaux constituent d'autant des appareils contemporains. Au XIX ${ }^{\circ}$ siècle, en effet, l'arrivée de la photographie a provoqué bien plus qu'un changement perceptuel. Elle a permis de mener des expériences sur la décomposition du mouvement (galop du cheval par Muybridge et décomposition du vol des oiseaux par Marey l'inventeur de la Chronophotographie, ancêtre direct du cinématographe). Par ailleurs, ravissant à la peinture sa fonction figurative, la photographie a orienté cette dernière vers la subjectivité du regard impressionniste. Les différents appareils de vision ont en effet une propension à objectiver la relation à l'image, renvoyant les dispositifs de représentation antérieurs et plus précisément ici la peinture à la subjectivité de l'expression artistique. Introduisant la notion de vision objective ainsi qu'une nouvelle forme de réalisme de la représentation que nous pourrions appeler représentation-trace et qui témoigne par la captation d'une image optique de l'existence d'une scène la photographie va constituer un moyen et un terrain d'échange entre art et science. Comme le rappelle en effet Monique Sicard (1991) :

\begin{abstract}
«les images du scientifique Marey offrent à voir le "jamais vu", de scènes pourtant familières et ce nouveau regard influence les peintres. Certains n'hésitent pas à rectifier sur leurs propres tableaux la représentation des allures du cheval. Le "Nu descendant un escalier" (1912) de Marcel Duchamp, le "Chien tenu en laisse" (1912) de Giacomo Balla font explicitement écho aux images d'Etienne-Jules Marey; la démarche chronophotographique se retrouve dans plusieurs tableaux des futuristes italiens qui vers 1910 s'intéressent à la représentation du mouvement»?
\end{abstract}

Saisie par les altérations et métamorphoses qu'apporte le traitement numérique, les images possèdent en elle une part de virtualité qui s'offre à l'exploration et à l'usage :
«les images virtuelles ne sont jamais seulement des images, juste des images, elles possèdent des dessous, des derrière, des en-deçà et des au-delà, elles forment des mondes. On n'en fait pas le tour. Il faut les explorer sans fin, comme des images mais aussi comme des idées, car elles ont partie liée avec les modèles. Elles peuvent nous faire remonter, de manière tangible, matérielle, aux idées qui les engendrent» (Quéau, 1993, introduction).

Enfin, précisions que pour l'artiste Miguel Chevalier ${ }^{8}$ l'image peut devenir un matériau artistique soumis à variation et généré par les dispositifs interactifs. Dans Autres natures ${ }^{9}$

\footnotetext{
${ }^{5}$ Les modalités d'apparition de cet espace opèreraient, selon Jean-Louis Boissier selon un mode constructiviste au cours duquel la perspective est calculée par l'ordinateur.

${ }^{6}$ Pour Jean-Louis Boissier, l'actualisation de l'image-relation relève du jeu.

${ }^{7} \mathrm{Nb}$ : ce texte de introductif de l'ouvrage de Monique Sicard Images d'un autre monde. La photographie scientifique n'est pas paginé.

${ }^{8}$ Pour plus de détails sur Autres natures et $18 \mathrm{~h} 39$ voir notre thèse de doctorat (Morelli, 2000).
} 
l'image fait en effet à la fois l'objet d'une présentation et de métamorphoses. Ces mutations prennent la forme d'une déconstruction suivie d'une recontextualisation permanente de l'image que l'artiste considère comme matière (Figure 1$)^{10}$. L'ensemble des travaux de cet artiste consiste en effet à déstructurer ses images afin d'en produire de nouvelles. Si les images de Miguel Chevalier sont à la fois éphémères et évanescentes, c'est parce que le traitement informatique peut créer le mouvement et ainsi les rendre virtuelles, en devenir. Toute nouvelle actualisation des images produites par les dispositifs que Miguel Chevalier conçoit est alors pour lui, à la fois source de complexification de sa démarche et de déclinaison de ses recherches plastiques l'amenant à penser l'image à la puissance image.
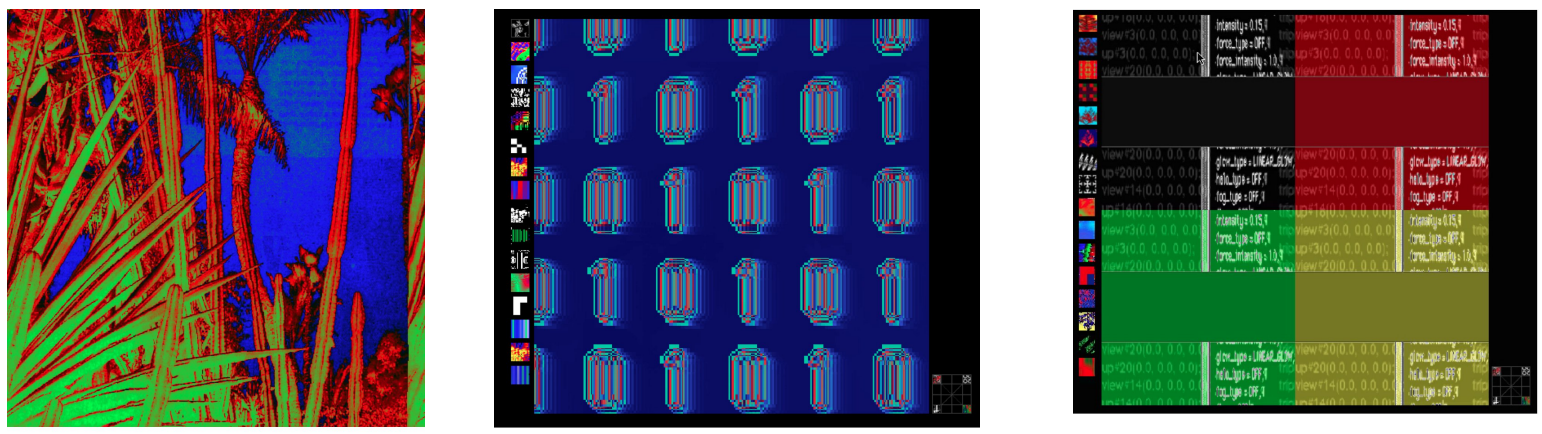

Figure1 - Autres natures, Miguel Chevalier 1996.

De façon plus globale, « perméables et parfois même altérables », les images s'enrichissent, à travers l'interactivité, d' "une dimension opératoire» offerte "à des expérimentations artistiques et à des pratiques médiatiques » (Fourmentraux, 2017 : 42). Lorsqu'elle s'exprime en ligne, cette dimension interactive produit des effets tant par la modalité avec laquelle l'image se donne à voir (la présentation ou la re-présentation) qu'au niveau de l'énonciation qu'elle formalise. Prise comme énoncé, l'image ne se limite plus à transmettre un signifié, elle «fait quelque chose » (Bianchini, 2012 : 139) et en retour devient un signifiant qui génère un nouveau signifié. L'image peut donc être également pensée comme interface narrative voire comme dispositif conversationnel.

\section{L'image comme interface narrative}

L'image ne se contente plus aujourd'hui de représenter ou de figurer le visible ou l'invisible. Elle devient de plus en plus l'interface à partir de laquelle vont s'exercer différentes formes d'interactions, l'image interface étant avant tout un dispositif frontière où se rencontrent et se négocient les intentions de l'auteur et de l'usager. Dans l'œuvre interactive multimédia 18h39 (Cédérom, 1997, Flammarion) Serge Bilous, Fabien Lagny et Bruno Piacenza invitent à la consultation d'un « instant» photographique $(18 \mathrm{~h} 39)^{11}$. Leur dispositif multimédia utilise une

\footnotetext{
${ }^{9}$ « Autres Natures est une série d'œuvres sur la nature et l'artifice, influencées par mon séjour au Japon à Kyoto. Il s'agit de la suite des serres botaniques Baroque \& Classique et également des premières œuvres interactives et totalement numériques. Le grand verre/nature liquide est constituée d'algorithmes, qui génère des images en perpétuel devenir. Ces œuvres virtuelles ne se déroulent plus de manière linéaire comme une bande vidéo, elles abolissent la fixité de l'image, et tendent vers l'immatérialité. Ces créations en 2D installées sur le disque dur d'un ordinateur évoluent en temps réel et leurs mouvements peuvent être modifiés à tout moment par le public grâce à une souris. Une nouvelle tension entre forme et informe, figuration et "dé-figuration" cristallise cette nouvelle possibilité de la représentation de l'inachevé et de l'infini. ». Accès http://www.miguelchevalier.com/fr/autres-natures-0. Consulté le 8 décembre 2016.

${ }^{10}$ Une barre d'outils s'offre à l'interacteur, à gauche de l'image. Seize possibilités de transformation de l'image sont proposées sous forme d'icônes à cliquer (voir Figure 1).

${ }^{11}$ «Que s'est-il passé à $18 \mathrm{~h} 39$ ? », question explicitement posée et inscrite sur la boite contenant le cédérom éponyme.
} 
interface fortement et ouvertement inspirée de la vision « machinique » présentée dans le film BladeRunner de Ridley Scott (1991). Le spect-acteur décortique une scène illustrée par une mystérieuse photographie en noir et blanc mettant en scène trois personnages : une femme à genoux tentant d'esquiver un coup porté par un homme debout, face à elle, et derrière lequel se trouve un inquiétant troisième personnage barbu, armé d'un fusil (Voir Figure 2).

La pléthore d'informations proposées dans $18 h 39$ invite à fouiller l'image et un ensemble d'informations textuelles, sonores, audiovisuelles et iconographiques ${ }^{12}$ afin d'en découvrir le sens. Les modalités d'avancée dans l'image sont prédéfinies par les auteurs. Il ne s'agit pas à proprement parler d'une immersion dans un espace 3D car le dispositif ne permet pas de s'immerger dans l'image afin de choisir son point d'observation. Il ne s'agit pas non plus de réalité virtuelle, la scène est en effet observée à travers la consultation d'éléments émanant d'une banque de données d'images photographiques. Leurs angles de vue, leurs cadrages diffèrent de manière à donner l'illusion d'une présence panoptique. L'avancée dans l'image est de plus, orientée, le zoom est dévié selon une direction imposée par les artistes. Les paramètres de prise de vue, les enchaînements ainsi que les modalités d'affichage de ces documents sont imposés par les auteurs (Figure 2).

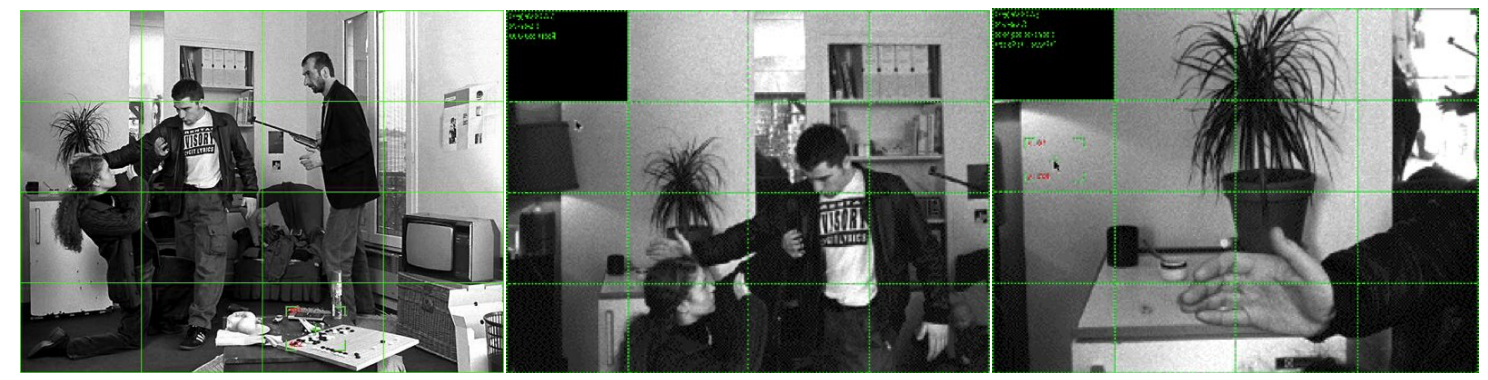

Figure 2- 18h39, Serge Bilous, Fabien Lagny et Bruno Piacenza - 1997.

Le spect-acteur est ainsi placé au centre d'un labyrinthe d'informations visuelles et textuelles qu'il ne peut ni collecter, ni mettre en rapport les uns avec les autres, si ce n'est en les mémorisant.

Autre forme de contrainte imposée, la progression dans les fouilles archéologiques de l'image ne peut s'effectuer que dans un seul sens. Il est impossible de revenir au niveau précédent sauf en de rares occasions et au bout de la navigation on revient toujours à l'inquiétante image initiale (Figure 2). L'avancée s'effectue donc à sens unique. Pour revenir à une position antérieure, il faut dans la plupart des cas retourner à la position initiale (niveau 0) sauf dans deux types de cas précis.

Nous avons affaire ici à trois types de mondes. Le premier monde est factuel. Il met en scène le spectateur en quête d'éléments à qui il donne un ensemble d'informations lui permettant d'expliquer, en la fouillant grâce à l'ordinateur, une photographie énigmatique. Le second monde est un monde de feintise qui, par l'accumulation de détails, de documents iconographiques (photos, vidéo, planches,...), d'outils de simulation qu'il procure crée, chez le spectateur, l'illusion d'être placé en situation d'analyse scientifique (intégrée au programme informatique) de l'instant photographique d'analyse. La feintise relève à la fois d'une parodie de dispositif ${ }^{13}$ puisque le programme mobilise plusieurs sortes de simulations ainsi que d'une série d'éléments d'identification qui rendent probable le monde dans lequel

\footnotetext{
${ }^{12}$ Relevés d'empreintes effectués sur la « scène de crime », banques d'images photographiques et audiovisuelles permettant d'apprécier la scène sous une multitude d'angles, tableau d'identification anthropométrique de Bertillon...

${ }^{13}$ Il s'agit d'une feintise énonciative (Cf. Jost, 1995).
} 
nous plonge le cédérom, notamment par l'ensemble impressionnant d'éléments concrets (photographies, vidéo) accessibles autour de la scène photographiée et par les messages (pseudo) scientifiques permanents. Le troisième monde est fictionnel puisque les trois protagonistes sont des acteurs et que tous les documents ont été filmés en studio. Ce monde se prolonge par la construction progressive d'un méta-récit par le spect-acteur chez qui l'absence de réponse à la question initiale fait naître un désir de narration (cf. Weissberg, 2000).

Remarquons par ailleurs que le mode dialogique qui saisit l'image à travers les réseaux numériques introduit une rupture par rapport à la communication «mass-médiatique », régime qui par principe veille à assurer, aux messages transmis par l'émetteur au récepteur ni dégradation physique ni dégradation sémiotique ( $c f$. Couchot, 1998 : 155). Avec le Web puis les médias sociaux, s'installe un espace intermédiaire entre émetteur et destinataire tant l'exercice de l'interactivité perméabilise les positions réciproques et altère que le rôle et la position de chacun. Comme le montre bien la typologie d'œuvres du Net Art que dresse JeanPaul Fourmentraux (2005 : 90-107) l'œuvre interactive comporte une part d'indétermination laissant à l'interacteur une liberté d'intervention dont les conséquences peuvent se répercuter sur le résultat final. Dans les dispositifs (du Net Art) à altération, précise cet auteur, l'internaute intervient par transformation ou apport de données, dans le respect strict des règles et des procédures alors que dans les dispositifs à alteraction l'interaction entre les internautes opère de manière plus ouverte au gré des communications qui s'installent entre eux pour aboutir à la création d'une œuvre collective. Un mode dialogique saisit donc l'image et nourrit l'activité des réseaux numérique. Il est d'ordre conversationnel car de nos jours, au même titre que les textes et les objets audiovisuels qui parsèment la toile, l'image assume une fonction éminemment relationnelle et participe à l'avènement et à la généralisation de modalités conversationnelles.

\section{L'image comme dispositif conversationnel}

Au contrat de lecture, garant de la création et de l'entretien d'une relation forte entre les médias et leur(s) public(s), concept qui s'inscrit avec l'idée de marketing éditorial, créé en 1985 dans un contexte concurrentiel renforcé et qui permet de distinguer les positionnements et les identités spécifiques en fonction du titre de presse lu, succède, selon le linguiste JeanMaxence Granier (2001), un contrat de conversation.

Le contrat de lecture se propose d'instancier les destinataires, de construire un «public représenté » auquel les lecteurs / auditeurs / téléspectateurs sont invités à s'identifier et à cet effet procède par mise en scène des figures de l'émetteur (producteurs de contenus, éditeurs, publicitaires et marques) et du destinataire (le lectorat). Il inscrit les relations spécifiques entre émetteurs et destinataires dans un monde construit déterminé, un univers de référence dans lequel opèrent et au sein duquel sont interprétées la production et la réception (chaîne communicationnelle). Rappelons que très vite, et bien avant le numérique, les médias ont introduit et cherché à développer toutes formes d'interactions avec leur(s) publics :

- le courrier des lecteurs, le recours à un organisme qui centralise les appels téléphoniques, établit une synthèse et identifie des cas particuliers qui seront cités en direct, pendant des émissions (SVP 11-11);

- avec le développement de la téléphonie mobile l'usage de SMS et avec l'avènement des réseaux sociaux en ligne la mise en place de dispositifs d'interactivité asynchrones comme les mots dièses (\#haschtags) ou synchrones et distribués sur plusieurs supports comme le double écran (Tablette ou smartphone et télévision).

Avec le contrat de conversation se développent des formes de médiation cadrées par soucis de structurer la temporalité sociale des échanges. L'asymétrie qui contraint les modalités de 
feedback peut aller jusqu'à empêcher le contrôle de l'information par le lecteur. Remarquons que ce retour en force de la médiation est particulièrement présent dans les formes postérieures au Web 2.0 qui installent un modèle communicationnel remettant en cause la position en surplomb des médias traditionnels sur les conversations. Soulignons également que souvent « le sujet de conversation fonde les groupes et les communautés d'intérêt sur le Web» (ibid. : 169). Avec le contrat de conversation se tissent donc des pratiques conversationnelles qui ne cessent de s'intensifier. Il n'est plus rare en effet de lire des commentaires portant sur d'autres commentaires.

Comment se construisent et s'articulent les activités discursives qui donnent vie à la pulsation que connaît l'Internet contemporain ? Penser la cartographie des actions à partir de lignes de fuites ne suffit pas tant le numérique est désormais siège et moteur d'échanges démultipliés. Fort de ce constat, l'architecte et artiste Olivier Auber ${ }^{14}$ invite à penser en termes de perspective numérique, approche

«fondée, non pas sur des points de fuite (...) mais sur des codes de fuite, de nature numérique, biologique, génétique, voire quantique, capables d'organiser des formes de commutation acentrée, synchrone ou asynchrone, à l'intérieur de la communauté humaine. ».

Cette proposition conceptuelle est en phase avec le développement, au sein des réseaux numériques, de dispositifs de pensée collective et connective (De Kerkhove, 1999) sur écran. Leur prolifération contribue, par ailleurs, à la formation et à la mise en visibilité de communautés d'intérêt ou d'appartenance ${ }^{15}$ opérant principalement sur un mode conversationnel. Dans l'espace public reconfiguré par les réseaux socionumériques, les frontières traditionnelles entre émission et réception deviennent alors de plus en plus poreuses, ce qui a pour effet de transformer l'écoute en participation. S'échangent alors, précise JeanMaxence Granier, des prises de parole qui s'exécutent sous les yeux de tous. De nouveaux rapports à l'information s'installent. Traditionnellement dévolu à la communication, le principe circulation de messages à travers différents canaux reliant destinateur(s) et destinataire(s) est sérieusement bousculé par la réciprocité des échanges, et l'influence croisée des paradigmes de la co-construction et de l'extimité (Tisseron, 2011) qui saisit l'information. $\mathrm{Au}$ sein des réseaux, fréquence et rapidité dans les échanges se conjuguent participant d'une construction discursive progressive et réciproque. Pour nommer cette situation communicationnelle inédite propre aux systèmes dialogiques, Edmond Couchot $(1998,156)$ propose la notion de commutation. Pour cet auteur en effet,

« il n'y a plus communication au sens strict, entre un énonciateur et son destinataire mais commutation, plus ou moins instantanée, entre un récepteur devenu émetteur, un émetteur devenu (éventuellement) récepteur et un "propos" flottant qui à son tour émet et reçoit, s'augmente ou se réduit. Le sens ne s'engendre plus par énonciation, transmission et réception, alternativement, mais par une hybridation entre l'auteur, le propos véhiculé par la machine (ou le réseau) et le destinataire. »

Ce type de co-construction n'opère toutefois pas uniquement sur le principe de la collaboration car, comme le précisent Erik Bertin et Jean-Maxence Granier (2015), le Web 2.0 rend accessible des possibilités de production et de diffusion de significations. S'installent alors des formes de concurrence entre les propositions qui circulent dans l'espace de communication, lesquelles présentent désormais une composante évaluative articulant dimension qualitative ${ }^{16}$ et dimension quantitative ${ }^{17}$.

\footnotetext{
${ }^{14} \mathrm{Http}: / /$ perspective-numerique.net/. Consulté le 14 novembre 2016.

${ }^{15}$ Pour Max Weber (1921) il convient de différencier la «communalisation », communauté subjective fondée sur le partage entre ses membres d'un sentiment d'appartenance, de la « sociation », communauté objective fruit d'un compromis [Aussgleich] ou d'une coordination [Verbindung] d'intérêts motivés rationnellement.

${ }^{16}$ Commentaires, arguments formulés...

${ }^{17}$ Nombre de « like », nombre de vues, nombre de commentaires, notation (satisfaction).
} 
Alors que le mode conversationnel saisit l'image, qu'elle soit montrée, échangée ou discutée cette dernière ne se contente toutefois pas de constituer, de déclencher ou d'illustrer le sujet de conversation. Elle peut désormais constituer l'interface à partir de laquelle s'établit et s'entretiennent les discussions en ligne. Le développement des conversations numériques s'appuie en outre sur la montée en puissance de l'usage des dispositifs communicationnels et bénéficie de la mobilité de la connectivité généralisée et de l'engouement des particuliers pour l'adoption de supports nomades (smartphones, tablettes).

Les conversations en ligne ont pour particularité de comporter en elles une potentialité d'ouverture susceptible d'échapper au contrôle des interlocuteurs car au final l'émetteur, le récepteur et le message lui même peuvent s'émanciper de la situation initiale. Émetteurs et récepteurs sont placés en effet au sein de différents réseaux comportant des membres dont chacun peut en effet mal contrôler l'activité. Le message en lui même est soumis au principe de "persistance réticulaire" phénomène que nous nommons ainsi par analogie avec la persistance rétinienne qui affecte la vision humaine incapable d'oublier instantanément les images qui imprègnent la rétine de l'œil, inertie physiologique sur la base de laquelle fut inventée la projection cinématographique. Mise en ligne, l'information gagne en autonomie du fait de l'entre-maillage des réseaux et donc les multiples interconnections entre membres de chaque communauté d'internautes mais aussi et surtout par l'action calculée et automatique dont le Web est le siège due aux dispositifs de quantification et de qualification des contenus en ligne (robots, référencement...) et la conséquence indirecte de la prolifération de sites miroirs qui dupliquent les contenus afin de fluidifier les échanges et limiter les temps d'attente pour l'accès à l'information en ligne ainsi que l'archivage temporaire ou définitif d'une partie des sites web visités (mémoire cache, archivage automatique ou programmé des informations obtenues par syndication de contenus, copies d'écrans). Pour qualifier cet état d'indétermination qui saisit la finalité des informations mise en ligne nous opterons pour le modèle de la viralité informationnelle qui saisit désormais les images.

\section{Images et viralité}

Parler de viralité informationnelle signifie s'engager dans une analyse de la contagiosité de l'information comme étant une action intentionnelle ou une résultante d'effets perlocutoires, voire comme envie de partager l'information et comme besoin de s'exprimer à partir d'elle et de converser avec ses pairs ou avec différentes communautés actives en ligne. La viralité peut aussi témoigner d'une créativité foisonnante qui s'exprime en ligne. Voyons à ce propos à quels acteurs sociaux et à quels processus créatifs nous avons affaire.

\section{Des acteurs de la viralité sur le Web et les réseaux sociaux}

Selon son principe fondateur, l'internet est constitué en puissance, d'une infinité de points de régénération des messages puisque, tout récepteur devient potentiellement émetteur, ce que les réseaux sociaux numériques contribuent à banaliser. Toute action (commentaire, marquage, mise en ligne) fait en effet l'objet d'une alerte adressée aux membres de la communauté virtuelle d'appartenance. Via l'usage d'hashtags, l'information peut, de plus, être regroupée, conférant à l'ensemble des contributions partageant un même hashtag une certaine visibilité. Cette pondération calculée par les dispositifs socio-numériques (tendances) n'échappe pas à la « sagacité » des moteurs de recherche et notamment des web-crawlers, à savoir les robots qui procèdent au repérage et la collecte des pages web.

Conçu sur le modèle du rhizome sans origine et sans finalité unique, le réseau des réseaux est a-centré et a-géographique ce qui complique d'autant la tâche des personnes chargées de soigner la e-réputation d'un individu ou d'une marque, à savoir la résultante des conversations numériques générées à partir d'informations produites et diffusées par l'individu ou la marque 
et les échanges verbaux entre internautes. Or la viralité qui saisit l'image bénéficie d'un goût affirmé par les internautes pour la mise en circulation et le partage massif d'informations reprises et métamorphosées sur la toile (Jeanneret (2008). Cette créativité qui se développe désormais avec l'intensification des conversations numériques crée, au sein de la toile des « chambres d'écho » ou persistent, malgré la persévérance des chargés de communication et gestionnaires de communautés en ligne des scories informationnelles. Le droit à l'oubli prend alors des tours inattendus et consiste moins en une suppression totale et définitive des contenus jugés obsolètes, déplacés ou inopportuns dont l'effacement complet est impossible qu'une stratégie de contournement visant à diminuer de manière drastique la pondération donc la visibilité des informations malveillantes. D'une certaine façon on se retrouve dans la logique même de l'aïkido, art martial visant à réduire les tentatives d'agression à néant par l'utilisation de la force de l'adversaire, et mieux de son agressivité donc de retourner contre lui sa volonté de nuire. Ici, il s'agit, non pas de vaincre les web-crawlers, mais de s'appuyer sur les caractéristiques fonctionnelles des algorithmes afin de renvoyer les effets dans les profondeurs du web, donc d'opacifier les informations pour moteurs de recherche les noyer dans la masse donc les invisibles.

La viralité s'organise à force de reprises et de traduction de l'image que certains vont recadrer, légender de manière à s'en approprier la force suggestive et à la recontextualiser à dessein. Parmi la chaine des acteurs sociaux qui vont partager l'image distinguons les influenceurs, individus disposant d'un très forte reconnaissance faisant d'elles des prescripteurs au sein des communautés dans lesquelles elles s'expriment. Cette reconnaissance relève de la notion de capital social telle que la définit Pierre Bourdieu (1980 : 31) :

\footnotetext{
« Le capital social est l'ensemble des ressources actuelles ou potentielles qui sont liées à la possession d'un réseau durable de relations plus ou moins institutionnalisées d'interconnaissance et d'interreconnaissance ; ou, en d'autres termes, à l'appartenance à un groupe, comme ensemble d'agents qui ne sont pas seulement dotés de propriétés communes (susceptibles d'être perçues par l'observateur, par les autres ou par eux-mêmes) mais sont aussi unis par des liaisons permanentes et utiles ».
}

Les influenceurs peuvent être classés selon le type d'activité dominante qu'ils exercent sur la toile $^{18}$, critère que nous croisons avec le type d'identité qui se construit à travers les réseaux sociaux. D'un point de vue strictement technique on peut isoler trois grandes catégories d'influenceurs ( $c f$. Giorgio Nova) : les « chasseurs, explorateurs \& cueilleurs d'information » qui opèrent souvent dans l'urgence et de façon continue, voire interrompue ; les " sages », auteurs qui affectent de s'abstraire de la pulsation du web afin de prendre le temps de l'analyse et de l'écriture afin de tempérer et renforcer leurs propos et les essayistes, pour lesquels les thématiques empruntées et les sujets sont avant tout une occasion de mettre en avant des qualités rédactionnelles et discursives; et les «extrapolateurs », personnages inventifs qui utilisent les réseaux pour faire circuler leur pensée. Les premiers sont avant tout des sentinelles, des dénicheurs d'information dont l'activité principale consiste à parcourir le Web et les réseaux sociaux, et afin d'être toujours informés. Ils s'abonnent à toutes sortes de contenus et multiplient les liens avec tous types de décideurs afin de soigner la fraicheur de l'information qu'il vont relayer au plus vite dans leurs réseaux d'influence. Les deuxièmes sont soit des figures historiques du Web, soit des personnalités reconnues dans leurs milieux professionnels d'appartenance. Ce sont des «tisserands » dont la préoccupation première est la formalisation du sens à partir des informations recueillies. La forme doit pour eux toujours rester en lien avec le fond. Les troisièmes correspondent d'une certaine façon aux « chamanes» de la typologie de bloggeurs de Giorgio Nova, personnages qui mettent leurs

\footnotetext{
${ }^{18}$ Nous reprenons volontiers ici en l'adaptant, l'approche typologique de la blogosphère telle que l'entreprend le blogueur italien Giorgio Nova (Morelli, 2008 : 174-175).
} 
très grandes capacités d'extrapolation au service d'une forte imagination et d'un art consommé de la provocation. Ces pamphlétaires peuvent à l'occasion s'affirmer être de redoutables "snipers" prompts à concentrer leurs attaques et à mobiliser leurs qualités rédactionnelles et expressives ou artistiques pour des objectifs précis. Lorsqu'ils opèrent eu sein des réseaux sociaux ils se comportent en «trolls »s'activant afin de cultiver le doute ou attaquer frontalement et ad nominem des personnalités reconnues dans leur domaine par pur intérêt.

Ces influenceurs existent pour et par le public qu'ils sont en mesure de rassembler autour d'eux (nombre d'abonnés, de followers, nombres de vue de leurs épisodes en ligne...). D'une certaine manière ils se constituent comme media à part entière étant reconnus par leurs publics pour la qualité de leurs visions et de leurs jugements. Critiques avertis, évaluateurs professionnels ou journalistes spécialisés ils opèrent sur la toile en leur nom propre ou pour le compte de médias, de décideurs ou d'instances politiques.

La reconnaissance, pour ces influenceurs, peut venir des pairs ou des publics qu'ils ciblent et qui ne manquent pas de les citer et qui s'abonnent à leurs productions en ligne. Elle peut également être appuyée par les médias qui leurs ouvrent leurs colonnes en qualité de consultants dont la parole et la figure sont prisées. Experts-figures d'autorité, certains influenceurs se sont dorénavant installés de manière durable dans les médias. C'est notamment le cas des bloggeuses-mode qui apportent aux magazines qui leur offrent des chroniques récurrentes, outre leur signature amis également un public potentiel et des annonceurs, ce qui n'est pas négligeable en ces temps de crise que traversent les médias. Pour connus qu'ils puissent être ces influenceurs n'ont cependant pas le monopole de la transmission virale de l'image. Des relais ponctuels, sporadiques et occasionnels peuvent en effet émerger et, pour peu qu'un consensus se mette en place pour reprendre les propositions provoquer un effet boule de neige. Une proposition peut, par exemple, être reprise par des agents réplicateurs qui la transmettent dans leurs réseaux ou la relancent assortie de commentaires ou de retouches jusqu'à inonder ou saturer les réseaux. Reprise, déclinée, discutée, donc négociée, une proposition singulière peut alors monter en puissance et s'imposant devenir collective ${ }^{19}$.

\section{La viralité comme moteur marketing}

Grands pourvoyeurs d'images, les responsables marketing rapprochent volontiers les potentialités relationnelles et l'engouement que les internautes développent pour l'usage de supports nomades et conceptualisent le picture marketing ${ }^{20}$ pour lequel l'image devient interface et vecteur privilégié de la publicité ciblée et les échanges communicationnels.

L'émergence du Smartphone en 2007 renforce la place de l'image dans la communication interpersonnelle. «Likée », partagée, commentée l'image circule, chacun ayant facilement la possibilité de la relayer avec ou sans commentaire à ses contacts. Cette propension à voyager

\footnotetext{
${ }^{19}$ Voir notamment notre article au sein de cet ouvrage et dans lequel nous prenons l'exemple de la propagation de l'hashtag "\#JeSuisCharlie » peu de temps après l'annonce de l'attentat visant les locaux du journal satyrique Charlie hebdo à Paris, le 7 janvier 2015.

${ }^{20}$ « Le picture marketing fait généralement référence à l'utilisation croissante des images ou visuels dans le domaine du marketing digital et notamment dans l'utilisation marketing qui est faite des réseaux sociaux. Étant souvent considérées comme plus efficaces et comme favorisant l'engagement, les images sont ainsi de plus en plus utilisées dans la communication sur les réseaux sociaux. Cet usage du picture marketing se fait sur les réseaux sociaux «généralistes » et professionnels (Facebook, Twitter, LinkedIn) mêlant les différents types de contenus (image, texte, vidéo), mais également sur ceux basés principalement sur l'utilisation des images (Pinterest, Instagram). ».Source : http://www.definitions-marketing.com/definition/picture-marketing/. Consulté le 22 décembre 2016.
} 
à travers les multiples réseaux de connaissance ou d'intérêt confère à l'image un statut nouveau et offre aux annonceurs et aux agences de publicité d'énormes potentialités communicationnelles. Conscient du glissement des pratiques de connexion vers les solutions nomades certains annonceurs réclament désormais l'usage de Snapchat et Instagram plutôt que Facebook ou Twitter afin de capter l'attention des internautes et de déclencher l'acte d'achat ${ }^{21}$. Au constat de partage quotidien via les médias sociaux de millions de photographies, de vidéos ou d'infographies s'ajoutent deux autres facteurs. Le premier est ergonomique. L'écran des smartphones est, du fait de la taille des écrans en effet mieux adapté à l'image qu'au texte. Le second est culturel. La montée en puissance de l'internet mobile concerne avant tout un public jeune, la génération Y qui a grandi avec l'internet et les ordinateurs portables laisse sa place à la génération $\mathrm{Z}$ qui a grandi avec les écrans tactiles et l'internet mobile.

Le mobile marketing ${ }^{22}$ comme le picture marketing trouvent dans la communication politique à l'occasion de campagnes électorales un terrain favorable de mise en application. Si Barack Obama avait conquis en 2008 la nouvelle génération grâce à Facebook, Snapchat s'impose désormais comme le meilleur vecteur pour s'adresser à cette population. Cette application de partage de photos et de vidéos via plateformes mobiles donne à l'image échangée une double temporalité. Évanescente, l'image peut, tout comme le texte du chat être regroupée dans une histoire (Story) visible pendant 24 heures. Depuis l'introduction de "Memories », une fonctionnalité optionnelle, les images peuvent être sauvegardées dans un espace de stockage privé. Et c'est justement ce réseau social que l'équipe de campagne du camp démocrate a mobilisé pour l'élection présidentielle américaine de 2016. On estime en effet près de 70\% des utilisateurs actifs de Snapchat ont entre 13 et 25 ans et que 32\% des jeunes américains utilisent Instagram, réseau social au sein duquel près de 70 millions de photos sont échangées au quotidien.

\section{Conclusion}

L'image, avons nous vu, est non seulement objet et sujet de questionnements. Elle constitue l'interface et le support de l'action à distance au sein d'un enchevêtrement complexe d'informations mises en réseau. Ouverte à l'activité créative des internautes, elle alimente les conversations numériques, subissant, à travers l'échange toutes sortes de transformations plastiques.

Relayée et déclinée à travers les réseaux, l'image assume plusieurs fonctions au sein des conversations numériques. Symbolique, elle oriente les discussions. Injonctive, elle affecte de déclencher en retour une action ou une modification comportementale. Expressive, elle s'offre à interprétations. Prolifique, elle nourrit et stimule la créativité. Numérique, elle participe de la construction relationnelle de l'identité. Les dimensions déclarative, agissante et calculée qui fondent l'identité numérique (Georges, 2009) sont mises en pratique et en retour participent de la e-réputation des acteurs sociaux en présence et, pour chacun de sa reconnaissance en tant qu'individu agissant.

\footnotetext{
${ }^{21}$ Cette tendance a notamment été observée à l'occasion d'un stage de master effectué au printemps 2016 par une étudiante de Master, spécialité création de projets numérique (Université de Lorraine) en Californie.

${ }^{22}$ « Le marketing mobile regroupe l'ensemble des techniques marketing basées sur l'utilisation des terminaux mobiles dont notamment et principalement le smartphone. Dans une acceptation plus large, le marketing mobile peut également être défini comme l'ensemble des actions marketing entreprises à destinations d'individus en situation de mobilité. (...) à ses débuts, le marketing mobile était essentiellement basé sur l'émission ou la réception de SMS, mais son univers s'est considérablement élargi avec les versions mobiles ou responsives des sites web et le développement des applications mobiles qui représentent désormais l'essentiel du temps passé sur smartphones. ». Source : http://www.definitions-marketing.com/definition/marketing-mobile/. Consulté le 22 décembre 2016 .
} 
La viralité de l'image n'est donc pas nécessairement une finalité en soi et constitue avant tout un témoignage d'existence. Reprise de manière virale, l'image peut, en effet, être considérée comme trace d'appropriation sémantique et sémiotique des propositions mises en ligne, entre entre intention et réception/réappropriation/traduction et produit d'effets qui participent, pour chaque acteur de la transmission virale, de la reconnaissance de soi. À l'heure de sa diffusion virale, l'image agit, pour tout individu, dans chacune des trois dimensions qui fondent, selon Axel Honneth la reconnaissance de soi, à savoir : la sphère affective, de la passion qui produit la confiance en soi, la sphère juridico politique qui place l'individu dans un ensemble articulé de droits et de devoirs dans laquelle se développe le respect de soi, ainsi que la sphère sociale dans laquelle s'affirme le sentiment de sa propre valeur forgé par confrontation des capacités et qualités individuelles de sorte à cultiver l'estime de soi. Mieux, à travers son parcours à travers la toile l'image équilibrera-t-elle en retour la confiance, le respect et l'estime de soi, plus l'individu sera-t-il conforté dans son rôle de relai et donc plus stable et pérenne sera la diffusion de l'image.

Avec et contre cette reconnaissance se construit alors une « figure du soi », véritable guide d'action permettant à chacun de se positionner et de garder un cap malgré la foultitude de propositions, de situations et d'actes rencontrés en ligne, donc à se présenter comme individu singulier et/ou comme membre d'une communauté de valeurs ou d'intérêts. L'identité relationnelle qui s'élabore à travers activités en ligne permet alors d'affirmer une certaine « continuité transsituationnelle (...) du soi» (Rosa, 2005: 293) donc de formaliser et de garder présent, à travers les multiples situations et rebondissements que génèrent les activités en ligne, un sens précis et affirmé «de ce que l'on est et qui procure une capacité d'orientation et d'action » (ibid.), ce qu'Hartmut Rosa nomme identité situative et qui permet de lisser le rapport au passé, au présent et à l'avenir ${ }^{23}$, donc à s'affirmer et à se décrire par l'action et ses résultats.

\section{Références}

Bertin É., Granier J.-M., 2015, «La société de l'évaluation : nouveaux enjeux de l'âge numérique », Communication \& langages, 184, pp. 121-146.

Bianchini S., 2012, « La performation. Quand faire c'est dire », in : Fourmentraux J.-P. (dir.), L'ère postmédia. Humanités digitales et cultures numériques, Paris, Éd. Harmann, pp. 137162.

Boissier J.-L., 2004, La relation comme forme. L'interactivité en art. Genève, Musée d'art moderne.

Bourdieu P., 1980, "Le capital social. Notes provisoires », pp.31-34, in: Bevort A., Lallement M., dirs, Le capital social, performance, équité et réciprocité, 2006, Paris, La Découverte-Mauss.

Couchot É., 1997, «A arte pode ser ainda un relógio que adianta ? O autor, a obra ou espectador na hora do temporeal » (L'art peut-il être encore une horloge qui avance ? L'auteur, l'œuvre et le spectateur à l'heure du temps réel), Editora da UNESP, São Paulo, Brazil.

- 1998, La Technologie dans l'art. De la photographie à la réalité virtuelle, Nîmes, Éd. Jacqueline Chambon.

\footnotetext{
${ }^{23}$ Pour Hartmunt Rosa (2005 : 293), l'identité situative permet «l'association narrative minimale du passé, du présent et de l'avenir et des différents registres du sens et de fonction de la vie du sujet (...) [association qui] se conforme à des modèles narratifs qui ont eux-mêmes leur origine dans les contextes situationnels spécifiques ».
} 
— 2007, Des images, du temps et des machines dans les arts et la communication, Nîmes, Éd. Jacqueline Chambon.

De Kerkckhove D., 1999, « Penser à l'écran », Art Press hors-série. L'art et la toile, p. 85.

Debray R., 1996, « Pourquoi le spectacle?», Les Cahiers de médiologie, ${ }^{\circ} 1$, La querelle du spectacle. Accès : http://mediologie.org/cahiers-de-mediologie/. Consulté le 18 avril 2017.

Déotte J.-L., 2004, L'époque des appareils, Paris, Éd. Lignes/Manifeste, Léo Scheer.

Flichy P., 1995, L'innovation technique. Récents développements en sciences sociales. Vers une nouvelle théorie de l'innovation. Paris, Éd. La découverte.

Forest F, Virilio P., 1988, « La fin des certitudes. Entretien avec Fred Forest et Paul Virilio », Art Press n 122, pp. 14-16.

Fourmentraux J.-P., 2005, Art et Internet. Les nouvelles figures de la création. Paris, CNRS Éd.

— 2016, «Images interactives et opératoires », in: Fourmentraux J.-P. (dir.), Images interactives. Art contemporain, recherche te création numérique, Bruxelles, Éd. La Lettre vollée.

Georges F., 2009, « Représentation de soi et identité numérique. Une approche sémiotique et quantitative de l'emprise culturelle du web $2.0 »$, Réseaux, 154, pp. 165-193.

Gui Ekwa M., 1995, Aspects sociologiques,3,1, Mars 1995, pp. 4-9.

Jeanneret Y., 2008, Penser la trivialité. Volume 1. La vie triviale des êtres culturels, Paris, Éd. Hermes-Lavoisier.

Jost F., 1995, « Le feint du monde », Réseaux, 72-73, pp. 163-175.

Marin L., Varrier J., 2017, « Récit », Encyclopediae Universalis

Morelli P., 2000, Multimédia et création. Contribution des artistes au développement d'une écriture multimédia, Thèse de doctorat en sciences de l'information et de la communication, Université de Metz. Accès: Tome 1: http://docnum.univ-lorraine.fr/public/UPVM/Theses/2000/Morelli.Pierre.LMZ0012_1.pdf, Tome 2: http://docnum.univlorraine.fr/public/UPV-M/Theses/2000/Morelli.Pierre.LMZ0012_2.pdf.

— 2006, «Recherche d'une problématique : du théâtre (texte et/ou représentation) comme modèle conceptuel et analytique de l'interactivité dans l'écriture multimédia », in: Thé@tre et nouvelles technologies, Garbagnati L., Morelli P. (dirs). Dijon, EUD, Coll. Écritures, pp. 17-32.

— 2008, Blogs et médias, quels rapports aujourd'hui : essai de typologie », in : Meimaris M. \& Gouscos D. (Éds.), Enjeux et usages des Technologies de l'information et de la communication. Médias et diffusion de l'information : vers une société ouverte, Athènes : Gutenberg, pp. 169-178.

Quéau P., 1993, Le virtuel, vertus et vertiges, Sessel, Champ vallon INA.

Rosa H., 2005, Accélération. Une critique sociale du temps, Trad. De l'allemand par Didier Renault, Paris, Éd. La Découverte, 2012.

Sicard M., 1991, «Introduction et légendes », in: Sicard M., Images d'un autre monde la photographie scientifique, Paris, Centre national de la photographie.

Tisseron S., 2011, « Intimité et extimité », Communications, 88, pp. 83-91.

Virilio P., 1988, « La fin des certitudes », entretien avec Fred Forest, Art Press, 122, p. 14. 
Weber M., 1921, Économie et société, 1, Trad. de l'allemand par J. Freud, P. Kamnitzer, P. Bertrand, É. de Dampierre, J. Maillard, J. Chavy, Paris, Éd. Pocket, 1995.

Weissberg J.-L., 1988, « De l'image au regard», in: Vers une culture de l'interactivité, Actes du Colloque, Cité des sciences et de l'industrie La Villette, Paris, pp. 53-62.

— 2000, «Récit, geste et présence (à propos de $18: 39$ ) », Séminaire : L'image actée comme expérience, Université de Paris 8. Accès: http://hypermedia.univparis8.fr/seminaires/semaction/seminaires/txt99-00/1839Weiss.htm. Consulté le 9-12-2016. 